\title{
MEDIAN FOREHEAD FLAP IN RECONSTRUCTION OF THE CUTANEOUS MIDFACE DEFECTS FOLLOWING RESECTION OF BASAL CELL CARCINOMA
}

\author{
Reda A. Nofal* and Ibrahim A. Abdelbar **
}

\begin{abstract}
Objective: The current work attempted to assess the median forehead flap (MFF) for reconstruction of mild and moderate defects of the midface after resection of basal cell carcinoma (BCC).

Patients and Methods: A total of 20 patients with T1-T2 midface basal cell carcinoma (BCC) were included in this study. Patient's ages ranged from 60 to 70 years. According to the size of the postsurgical defects $>$ At Tanta Tumor Institute, the patients were categorized into two groups: Group I (GI) included 10 patients with mild defects ( $2 \mathrm{~cm}$ or less) repair using median forehead flap(MFF) and Group II (GII), included 10 patients with moderate defects (more than $2 \mathrm{~cm}$ ) repair using median forehead flap(MFF).The patients were prospectively evaluated by parameters such as; Flap viability (capillary refill time, bleeding on puncture with a needle and the color of the skin paddle) and esthetic results. Preparation of patients and planning of the surgical technique were necessary to determine the size of the defect were employed for reconstruction of all defects.
\end{abstract}

Results : On comparing the results of GI and GII, the flap viability, patient satisfaction and donor site morbidity were found to be $80 \%, 70 \%$ and $0 \%$ in GI compared to $60 \%, 60 \%$ and $20 \%$ in GII respectively (the overall results were: $70 \%, 65 \%$ and $10 \%$ respectively). Postoperative infection and partial wound necrosis, were diagnosed in one patient of GI (20\%) and was successfully treated with specific antibiotics and local measures; and in $2(10 \%)$ patients of GII that failed to conventional treatment and lost most of the flap (the overall partial and complete flap necrosis was $30 \%$ ). One patient (5\%) of G II showed temporary fistula formation, which disappeared few weeks postoperatively. We conclude that, the median forehead flap insures skin coverage, muscle bulk and good blood supply therefore; it can remain viable additional it is a feasible and reliable technique.

KEY WORDS: Median forehead Flap (MFF), Reconstruction, Midface Defects, Resection, carcinoma

* Associate Professor of Oral and Maxillofacial Surgery, Faculty of Dentistry, Al-Azhar University.

** Health Director of Tanta Cancer Center and head of Surgical Oncology Department 


\section{INTRODUCTION}

The basal cell carcinoma of the midface is one of the main causes of defects in the maxillofacial area. Therefore, a key point of research into the management of this type of tumour is how to restore the patient's cosmetic appearance and function while radically excising the tumour. and represents one of the most difficult reconstructive challenges in head and neck surgery ${ }^{(1,2)}$.

Reconstruction of such defects may be achieved in a variety of ways, including pedicle myocutaneous flapsandfree-flaps.Variousflaps weresimultaneously employed for the reconstruction of such defects following extirpation of malignant tumors including: regional flap and distance flap depends on the type of the surgical defect; i.e., its size, location, and the intrinsic properties of the regional flap ${ }^{(3,4)}$

Microvascular free flaps have found wide applications in reconstruction. The main disadvantages of this technique include donor site morbidity, longer operation time, necessitate special surgical and nursing skills and longer hospitalization ${ }^{(5,6)}$.

Reconstruction using free grafts has a high failure rate due to the lack of vascularization. Pedicle myocutaneous flaps including median forehead flap overcome the problems associated with free grafts by supplying their own vascularity and softtissue and has a shorter operating time compared to microvascular free flaps ${ }^{(7,8)}$.

The median forehead flap is still one of the most popular flaps for repair of full thickness defect of the midface, nose, the eyelid and the cheek. The midline forehead flap is a workhorse for reconstruction of large cutaneous midface and nasal defects, including from ala to ala. It is characterized by its dependability, consistent anatomy, robust perfusion pressure at the pedicle base, and excellent texture match $^{(9,10) \text {. }}$

The midline forehead flap design has the advantage of a donor site scar in the midline of the forehead rather than paramedian, which is often less conspicuous. Periorbital defects often present a surgical challenge because of the need to provide ocular protection, lacrimal drainage system patency, eyelid function, and aesthetics ${ }^{(11,12) \text {. }}$

Paramedian forehead flaps are used to repair large surgical defects on the nose. To create a flap with sufficient length and arc position to reach the inferior subunits, the pedicle is positioned to include the right or left supratrochlear vessels ${ }^{(13,14)}$.

There are classically five layers covering the forehead: skin, subcutaneous layer of fibrofatty tissue, muscular aponeurosis sheath, loose areolar tissue connecting the galea aponeurosis sheath to the fifth layer, and the deepest layer, the periosteum or pericranium ${ }^{(15,16)}$.

To overcome on the drawback of different methods in the reconstruction of the midface defects and to ensure a satisfactory functional and aesthetic result, good texture and color of the flap, the median forehead flap was introducing in this study. Outcome was evaluated using functional and cosmetic criteria.

\section{AIM OF THE WORK}

The ideal technique for reconstruction of midface defects is still an issue of controversy. The current work attempted to assess the median forehead flap(MFF) for reconstruction of mild and moderate defects of the midface after resection of BCC.

\section{PATIENTS AND METHODS}

This study consisted of 20 patients 12 Males and 8 females suffered from basal cell carcinoma(BCC) of the midface area. Patient's ages ranged from 60 to 70 years (mean 65 years). All patients underwent the surgical excision of the primary tumors with immediate reconstruction with median forehead flap(MFF). According to the size of the postsurgical defects, the patients were categorized into two groups: Group I (GI) included 10 patients 
with moderate defects ( $2 \mathrm{~cm}$ or less) repair using median forehead flap (MFF) and Group II (GII), included 10 patients with large defects (more than $2 \mathrm{~cm}$ ) repair using median forehead flap (MFF). The patients were prospectively evaluated by parameters including: flap viability (capillary refill time, bleeding on puncture with a needle and the color of the skin pedicle) and esthetic results.

Surgical technique: The tumour was excised with 5-mm clinically tumour free resection margin and send the lesion for histological examination. In case of residual tumour the procedure was repeated until no more. The defect is modified by existing defect is enlarged so that the resultant scars lie along the borders of aesthetic subunits. Enlarging the defect in the cephalad direction does not add additional length or morbidity to the forehead flap as showed in fig 1,2,3,4,5 and 6 .

Template Design and Flap Elevation: A precise template of the defect is made from a suture packet to shape the 3-dimensional nature of the target area. It is then transferred to the midline of the forehead for tracing, a flap, care must be taken to avoid damaging the blood supply ${ }^{17,18)}$

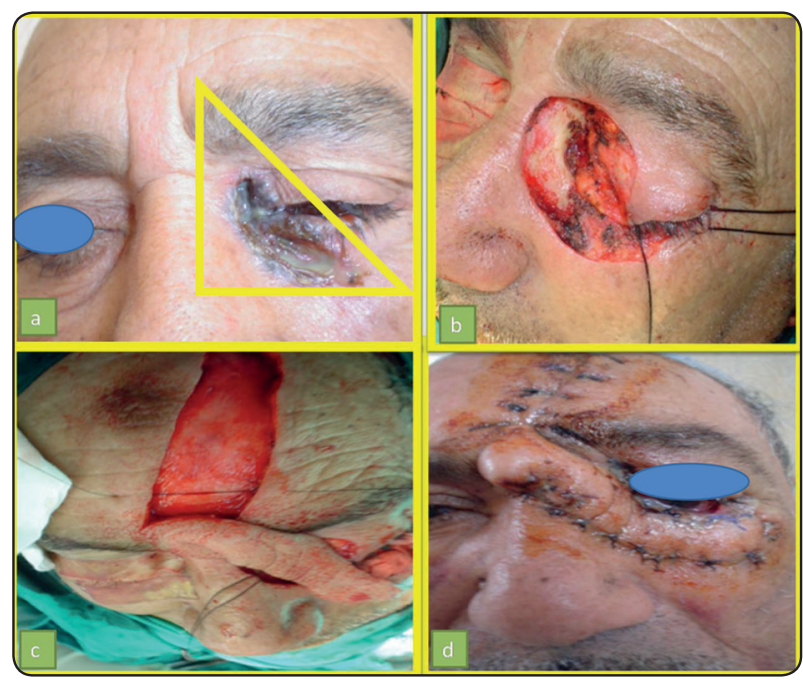

Fig. (1) Showing the preoperative view (a), mild defect after resection (b), MFF is dissected and cover the defect c), one week's postoperative view(d)

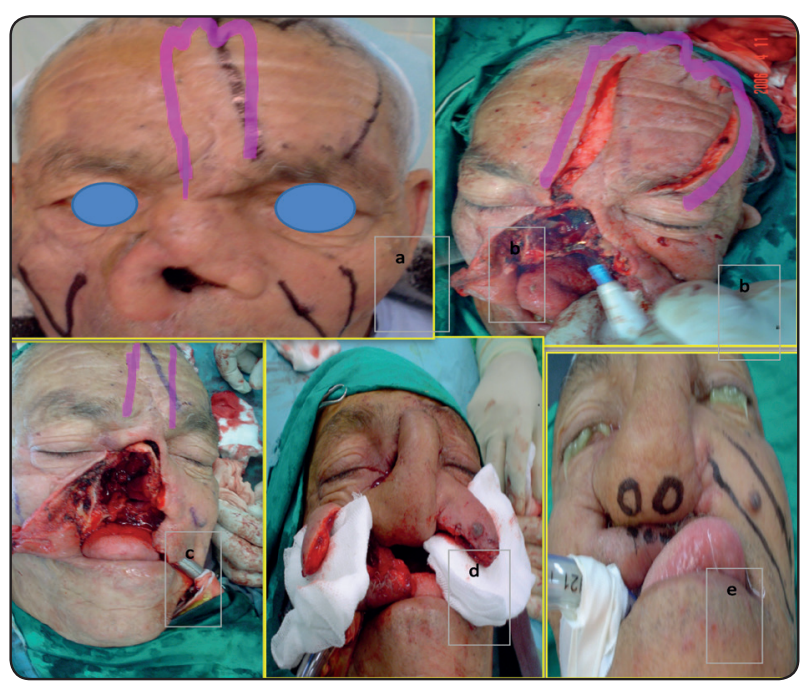

FIg. (2) Showing the preoperative marking of MFF (a)MFF dissection (b) the defect after resection (c), insert the MFF to form the nose (d), postoperative view(e)

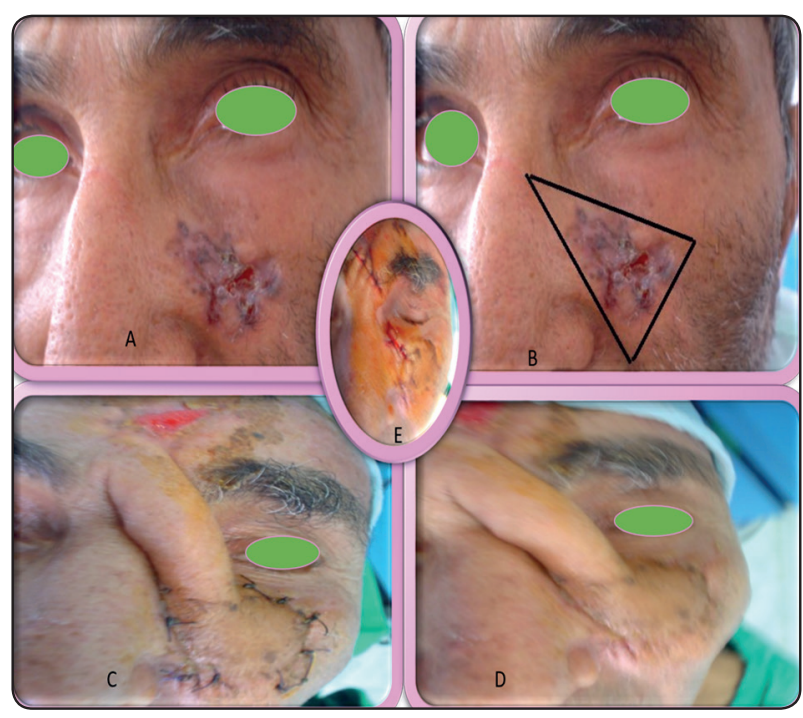

Fig (3) Showing the preoperative case (a), the marking before resection (b)MFF is in place 1 week (c) 3 weeks postoperative view(d\&f.)

Contralateral pedicle is designed to minimize rotation and kinking. The incision is made inside the tracing to match the defect, and elevation begins in the subcutaneous plane. Dissection then transitions to the subgaleal plane for the remainder of the flap and pedicle. At the pedicle base, just above the supraorbital rim, the periosteum is elevated and included with the pedicle, providing more length 
and rigidity to this region. The flap should then be planned so that the donor site can be closed near to the midline as possible. The length of the flap can be determined by stretching a suture from the distal-most aspect of the defect to the base of the flap. Blunt dissection, beginning 1 to $2 \mathrm{~cm}$ superior to the pedicle base, is used to elevate the pedicle in the subgaleal plane as showed in fig 1,2,3,4,5 and 6 .

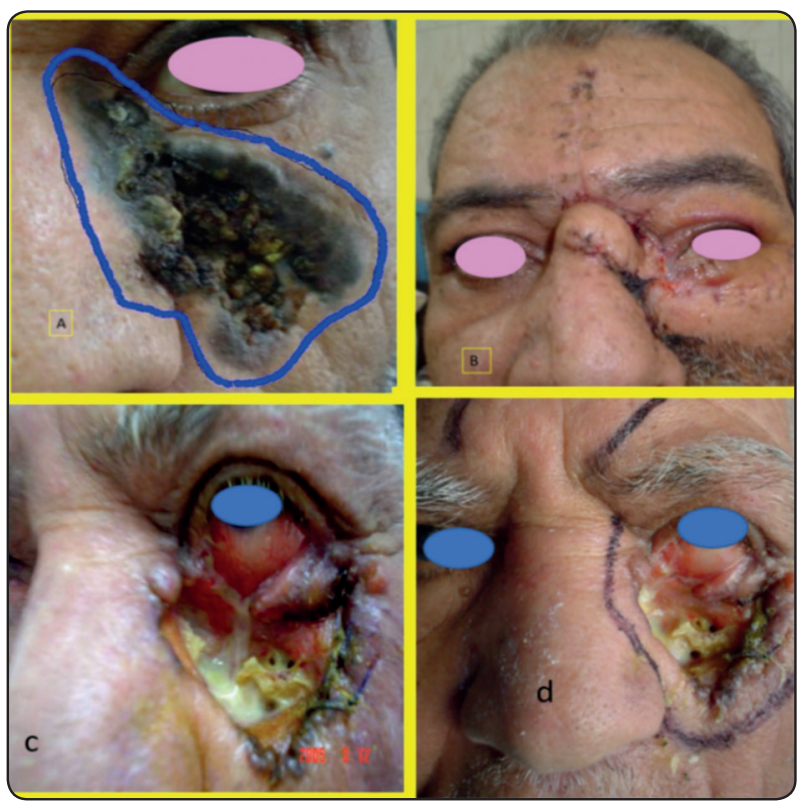

Fig. (4) Showing the preoperative marking before resection(a), one-week postoperative view (b) the preoperative view (c) the marking before resection(d)

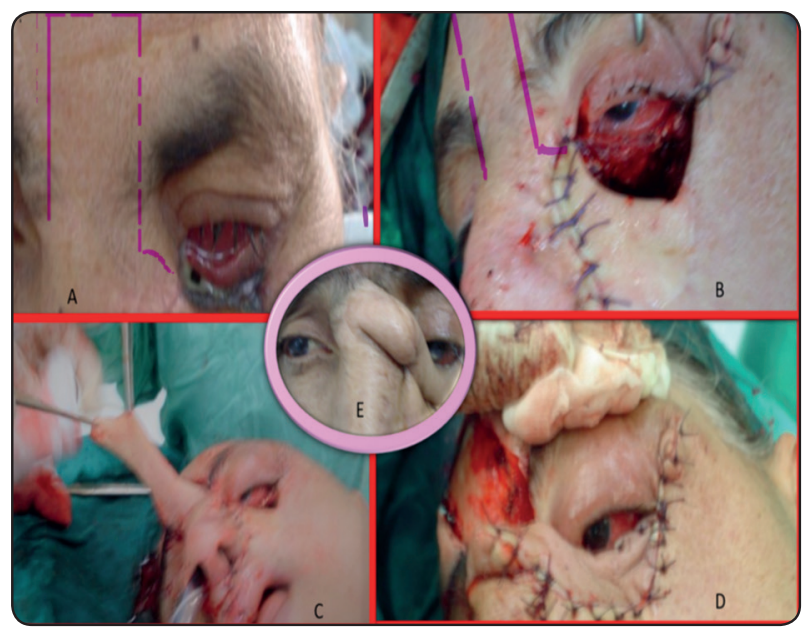

Fig. (5) Showing the preoperative marking (a), lesion is resected (b)MFF is harvested (c), insert the flap to form the lower eye lid (d), 1-week postoperative view(e)

\section{Forehead Flap Inset and Donor Site Closure:}

The forehead flap is secured with polydioxanone sutures and skin closed with Dixon sutures.

Flap monitoring: Observation of the flap by trained personnel was critical for the first entire 2 days postoperatively, and then the patient was followed up by clinical observation

The color of the skin paddle: provided additional information about the vascularity of the flap.

If the circulation in the flap was adequate, the color of skin would be normal or pink. If the arterial blood was compromised, the skin paddle would be pale in color. If the flap was congested due to obstruction, skin paddle color would be dark. Normal temperature of the skin paddle indicated adequate circulation in the flap. If the temperature of the skin paddle was less than body temperature, the circulation within the flap might be compromised. Capillary refill time provided information about the adequacy of the arterial supply as well as information regarding the presence of venous obstruction. Prolonged capillary refill indicated arterial problem, whereas, in venous obstruction, capillary refill was found to be faster than normal. Bleeding on puncture with a needle into the flap indicated good perfusion. During surgery, dermal bleeding was good evidence that blood flow through the flap had been established.

Criteria for evaluation of results: the parameters included: besides flap viability, operative complications, tumour recurrence, and restoration of cosmetic appearance and function.

Flap viability: Viability of the flap was assessed by the previously mentioned methods used for flap monitoring. According to the data given by these methods, flap was scored viable or non-viable ${ }^{\cdot(19,20)}$

Evaluation of aesthetics: Esthetic results were evaluated clinically and with the aid of medical photography. 


\section{RESULTS}

Twenty BCC patients (12 men \& 8 women) underwent immediate reconstruction after total resection of BCC from the midface. Their ages ranged from 60 to 70 years (mean was 65 years) and 60 percent were men. Patients were classified into 2 group: Group I (GI) included 10 patients with mild defects ( $2 \mathrm{~cm}$ or less), the lesion was resected and reconstructed using median forehead flap(MFF) and Group II (GII), included 10 patients with moderate defects (more than $2 \mathrm{~cm}$ ). the lesion was resected and repaired using median forehead flap(MFF). The twenty cases were treated by surgical removal of the primary lesions with $5 \mathrm{~mm}$ safety margin and reconstruction with MFF. The follow up period ranged from 6 to 28 months (mean 16 months) with no recurrences or mortalities.

TABLE (1) Most Commonly Involved Structures in Periorbital Midface Defects in G1\&11

\begin{tabular}{|l|c|c|}
\hline \multicolumn{1}{|c|}{ Structures } & No. of Patients & $\%$. Of patients \\
\hline Upper eyelid & 4 & 20 \\
\hline Medial canthus & 3 & 15 \\
\hline lower eyelid & 8 & 40 \\
\hline Left cheek & 2 & 10 \\
\hline Nasal sidewall & 3 & 15 \\
\hline
\end{tabular}

In $\mathrm{G} 1$, the most commonly involved structures included the medial canthus, lacrimal drainage system, upper and lower eyelids, and nasal sidewall as shown in table 1

G II, with larger defects, (average $5 \mathrm{~cm} \mathrm{x} 2 \mathrm{~cm}$ ) included 10 BCC cases (T2) completely affected the lower eyelid and cheek area and treated by removal of the primary lesion and reconstruction with MFF (Fig 4\&5).

The results of the flap viability, esthetics, donor site morbidity and postoperative complications of both groups are shown in fig 1,2,3,4,5,7,8 and 9 and table 1 .
TABLE (2) Showing the results of flap viability, patient esthetics, donor site morbidity and complications in both groups" Percentage and number of patients

\begin{tabular}{|c|c|c|c|}
\hline $\begin{array}{c}\text { Parameter } \\
\text { of the } \\
\text { assessment }\end{array}$ & $\begin{array}{c}\text { GI with } \\
\text { moderate } \\
\text { defects }\end{array}$ & $\begin{array}{c}\text { GII with } \\
\text { large defects }\end{array}$ & Overall \% \\
\hline Flap viability & $80 \%$ & $60 \%$ & $70 \%$ \\
\hline Esthetics & $70 \%$ (Good) & $60 \%$ (fair) & $65 \%$ \\
\hline Donor site & $0 \%$ & $20 \%$ & $10 \%$ \\
morbidity & $0(20 \%)$ & $1(20 \%)$ & $1(10 \%)$ \\
\hline $\begin{array}{c}\text { Fistula: } \\
\text { Necrosis: }\end{array}$ & $1(20 \%)$ & $2(40 \%)$ & $3(30 \%)$ \\
Infection: & $1(20 \%)$ & $2(40 \%)$ & $3(30 \%)$ \\
Flap failure: & $0(0 \%)$ & $2(40 \%)$ & $2(20 \%)$ \\
Recurrence: & $0(0 \%)$ & $0(0 \%)$ & $0(0 \%)$ \\
\hline
\end{tabular}

On comparing the results of GI and GII, as showed in fig 7, table $1 \& 2$ the flap viability, patient satisfaction and donor site morbidity(fig 8) were found to be $80 \%, 70 \%$ and $0 \%$ in GI compared to $60 \%, 60 \%$ and $20 \%$ in GII respectively (the overall results were: $70 \%, 65 \%$ and $10 \%$ respectively). Postoperative infection and partial wound necrosis, were diagnosed in one patient of GI (20\%) and was successfully treated with specific antibiotics and local measures; and in 2 (40\%) patients of GII that failed to conventional treatment and lost most of the flap (the overall partial and complete flap necrosis was $30 \%$ ). One patient (20\%) of G II showed temporary fistula formation, which disappeared few weeks postoperatively.

Fistulation, soft tissue necrosis, wound infection was found almost in all unsuccessful cases. Fistula formation with its serious sequelae of wound infection and/or abscess formation was diagnosed in the two cases of GII which underwent flap necrosis and failure.

Wound infection was reported in three cases; one of them (GI) was cured, while the other two flaps were found to be necrotic and failed to treatment. 


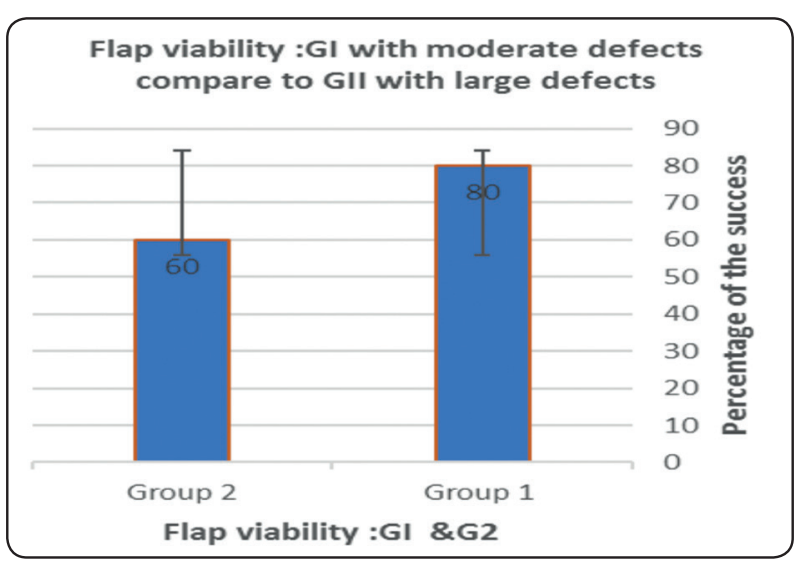

Fig. (6) Showing flap viability in G1 \&G2

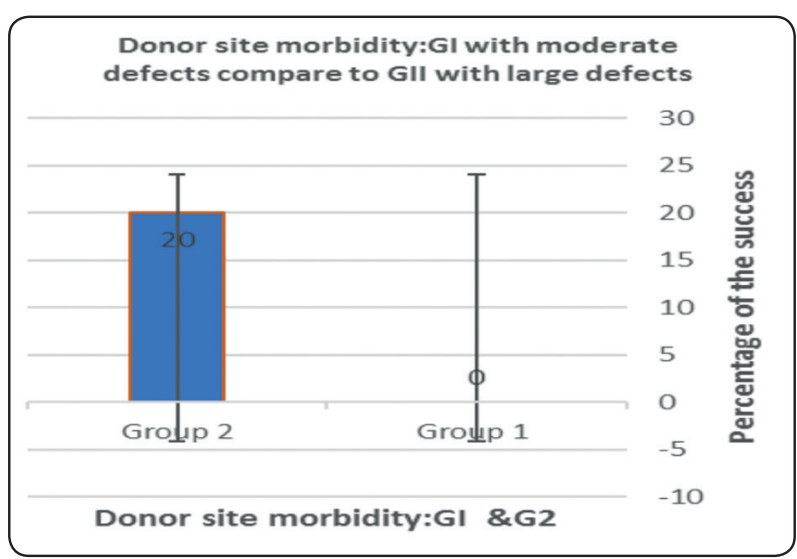

Fig. (7) Showing donor site morbidity in G1 \&G2 .

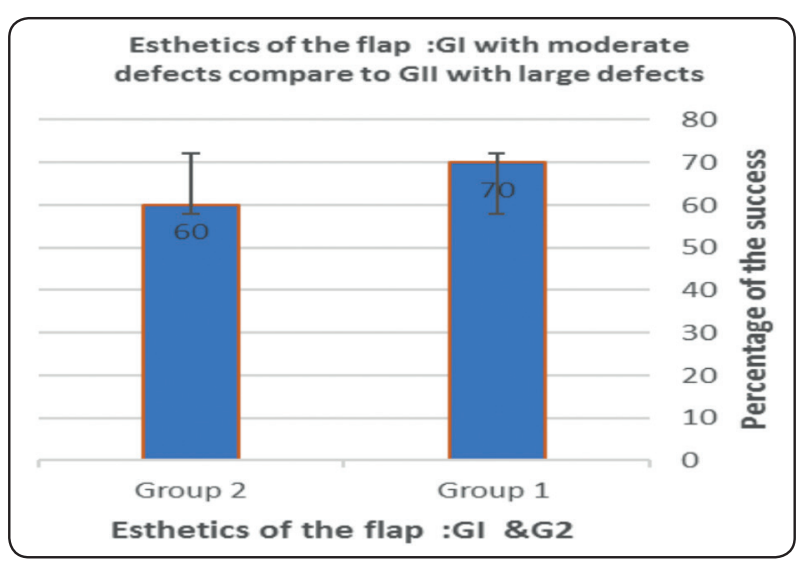

Fig. (8) Showing esthetics of the flap in G1 \&G2 .

\section{DISCUSSION}

The main objectives for head and neck cancer surgery are to eliminate the primary malignant tumor with adequate safety margins, to remove the involved or possibly involved cervical lymph nodes, to maintain and provide the best possible function and cosmetic results. Major excision and reconstruction for the face tissues, invariably interferes with these functions essential for normal living ${ }^{(15,16) \text {. }}$

Successful reconstruction requires a suitable fullness to achieve an acceptable contour; the intraoral lining requires a hairless flap; while the external face requires a flap with suitable thickness, texture, and color matching.

Even with the advent of microsurgical flaps, forehead myocutaneous flap persisted as the most widely used reconstruction technique by head surgeons. Therefore, it is considered as one of the main reconstructive techniques for large midface defects. $^{-(20)}$

Any improvement on the technique provides obvious practical implications due to its frequency of use. We found MFF dependable, reliable, esthetically and functionally accepted and has a long vascular pedicle of muscle that can easily reach the midface regions. The skin is supplied by musculocutaneous perforators, which are widely distributed over its surface. If the proximal musculocutaneous perforators are preserved, viable skin and fascia can be taken beyond the borders of the muscle.

Flap necrosis is a vital complication that may directly influence the result of the operation. In this study, a total of $15 \%$ (3 out 20) necrosis rate was recorded; the main cause of whole or partial necrosis of skin was the obstruction of venous drainage. Therefore, it is vital to broaden the tunnel of the flap and avoid compressing the flap pedicle. Various viability rates were reported in different 
studies; 50\% reported by Menick et al.,2105, 60\% by Okazaki et al., 2104; compared to $70 \%$ in the current study. In accordance to Roerich et al., 2009, the lower viability rates of pedicle myocutaneous flaps in the patients of GII may be attributed to the large size of the postoperative defects and preoperative systemic compromised of the primary tumor, which might affect the vascularity of the flaps.

Most of the maxillofacial surgeons agree on the need of primary reconstruction specially the midface defects to prevent the devastating aesthetic and functional sequelae. On comparing primary and secondary reconstructions, different reports showed that primary reconstruction has better chances in viability while they were equal in cosmoses and donor site morbidity. Furthermore, the use of tissue transfers either alone or in combination with other flaps allowed reliable one-stage reconstruction which significantly reduced the length of hospitalization.

Closure of a large donor site under excessive tension increases the risk of suture dehiscence and skin graft is too far from the edge being considered ideal. Alternatively, the use of adjacent flaps to close these large defects is an easy to employ technique, which produces good results and reduces the risk of complications associated with primary closure made under excessive tension. In the current study, primary closure of the donor site was successfully employed without major complications; however, it may be used along with other complementary techniques.

\section{CONCLUSION}

The median forehead flap insures skin coverage, muscle bulk and good blood supply therefore; it can remain viable additional it is a feasible and reliable technique.

\section{REFERENCES}

1. Park SS. The midline forehead flap in nasal reconstruction. Facial Plast Surg Clin North Am. 19(1):141-155, 2106

2. Menick FJ. Nasal reconstruction: seeking a fourth dimension. Plast Reconstr Surg. 2015;78(2):145-157.

3. Hughley BB, Park SS. Complications with forehead flaps in nasal reconstruction. Laryngoscope. 119(6): 1093-1099, 2016.

4. Neelam FW. Long-term results of blood flowand cutaneous sensibility of flaps used for the reconstruction of facial soft tissues. J Oral Maxillofac Surg. 2015;52:1247-1252.

5. Marsico RE Jr. Field guide to local flaps. Dermatol Clin $16: 65-74,2010$

6. Baker SR. Regional flaps in facial reconstruction. Otolaryngol Clin North Am. 23:925-946, 2011

7. FKonzo B. Use of skin flaps in dermatologic surgery of the face. J Dermatol Surg. 1:25-30, 2014

8. Menick FJ. Nasal reconstruction: forehead flap. Plast Re constrSurg 113:100-11, 2016

9. Menick Broad DG. Paramedian forehead flap reconstruction for nasal defects. Dermatol Surge ; 31:1046-52, 2011

10. Gladstone HB. Tissue oximetry for localized flaps. Oral presentation at the Annual American Society of Dermatologic Surgery Meeting; New Orleans, LA; 50(1): 90-99, 2011

11. Tripoli M, Toya F, Moschella F: Propeller flap for single-stage nose reconstruction in selected patients: supratrochlear artery axial propeller flap. Facial Plast Surg 30: 332-341, 2014

12. Morris SF, Nelligan PC: Perforator flaps: evolution, classification, and applications. Ann Plast Surg 50(1): 90-99, 2013

13. Manisha N, Shimizu Y, Shimizu R, Okabe K, Nakajima $\mathrm{H}$ : Alternative 1-step nasal reconstruction technique. Arch Facial Plast Surg 14(2): 116-121, 2012

14. Menick FJ: A 10-year experience in nasal reconstruction with the three-stage forehead flap. Plast Reconstrue Surg 109(6): 1839-1855, 2015

15. Menick FJ: Aesthetic and reconstructive rhinoplasty: a continuum. J Plast Reconstr Aesthet Surge 65(9): 1169-1174, 2012

16. Okazaki M, Ueda K, Sasaki K, Shiraishi T, Kurita M, Harii K. Ann Plast Surg 63(2): 167-170, 2014 
17. Griffin JR, Ansari M, Beran SJ, Potter JK: Nasal reconstruction beyond aesthetic subunits: a 15-year review of 1334 cases. Plast Reconstr Surg 114(6): 1405-1416, 2009

18. Skaria AM: The median forehead flap reviewed: a histologic study on vascular anatomy. Eur Arch Otorhinolaryngology 272(5): 1231-1237, 2015
19. Kazanjian VH. The repair of nasal defects with the median forehead flap: primary closure of the forehead wound. Surge Gynecology Obstet. 83:37-42, 1966.

20. Smith TL. The anatomic basis for the design of forehead flaps innasal reconstruction. Arch Otolaryngology Head Neck Surg. 118:373-379, 2011 